\title{
Edge bi-magic total labeling on signed necklace
}

\author{
R. Chitra, ${ }^{1 *}$ and S.P. Subbiah ${ }^{2}$
}

\begin{abstract}
Gracefulness and Skolem gracefulness on signed graphs are discussed in [6] and [7]. No other types of labeling are studied on signed graphs. Here we have introduced a new type of labeling namely, edge bi-magic total labeling on signed graphs and investigates such labeling on some classes of signed graphs.

An injective labeling $f: V \cup E^{+} \cup E^{-} \rightarrow\{0,1,2, \ldots, p+q\}$ on a signed graph $S(V, E, \sigma)$ is said to be edge bi-magic total if $f(u)+f(v)+\sigma(u v) . f(u v)=k_{1}$ for all $u v \in E^{+}$and $f(u)+f(v)+\sigma(u v) \cdot f(u v)=k_{2}$ for all $u v \in E^{-}$, where, $k_{1}>0$ and $k_{2}>0$. The positive magic weight of $f$ is $w^{+}=k_{1}$ and $w^{-}=k_{2}$ is the negative magic weight of $f$ on the signed graph $S$.

The positive upper strength $\left(S^{+}\right)$of a signed graph is equal to $\max \left\{w_{1}^{+}, w_{2}^{+}, w_{3}^{+}, \ldots, w_{m}^{+}\right\}$, where $w_{1}^{+}, w_{2}^{+}, w_{3}^{+}, \ldots, w_{m}^{+}$ are positive magic weights of $f_{1}, f_{2}, f_{3}, \ldots, f_{m}$ on $S$ respectively. The positive lower strength $\left(s^{+}\right)$of a signed graph is equal to $\min \left\{w_{1}^{+}, w_{2}^{+}, w_{3}^{+}, \ldots, w_{m}^{+}\right\}$.

Similarly the negative upper strength $\left(S^{-}\right)$of a signed graph is equal to $\max \left\{w_{1}^{-}, w_{2}^{-}, w_{3}^{-}, \ldots, w_{n}^{-}\right\}$, where $w_{1}^{-}, w_{2}^{-}, w_{3}^{-}, \ldots, w_{n}^{-}$are negative magic weights of $f_{1}, f_{2}, f_{3}, \ldots, f_{n}$ on $S$ respectively. The negative lower strength $\left(s^{-}\right)$of a signed graph is equal to $\min \left\{w_{1}^{-}, w_{2}^{-}, w_{3}^{-}, \ldots, w_{n}^{-}\right\}$. This is total in nature because all the vertices and edges are labeled. All positive and negative magic weights are always greater than or equal to zero, no negative weights are taken in to account.

In this paper, we introduce and investigate the edge bi-magic total labeling on signed necklace.
\end{abstract}

\section{Keywords}

Edge bi-magic total labeling, Positive magic strength, Negative magic strength, Signed Necklace.

\section{AMS Subject Classification}

$05 \mathrm{C} 78$.

${ }^{1}$ Department of Mathematics, The Madura College, Madurai-625011 Tamil Nadu, India.

2 P. G. and Research Department of Mathematics, Mannar Thirumalai Naicker College, Madurai-625004 Tamil Nadu, India.

*Corresponding author: ${ }^{1}$ rchitramphil@gmail.com

Article History: Received 21 December 2018; Accepted 11 February 2019

(C)2019 MJM.

\section{Contents}

1 Introduction .90

2 Edge bi-magic total labeling on signed graphs .... 91

3 Edge bi-magic total labeling on signed necklace .. 91

References .93

\section{Introduction}

Signed graphs form an interesting area in graph theory. Labeling of signed graphs is a new area. Edge bi-magic total labeling of signed graphs is attempted in this paper.

A labeling of a graph $G$ is a mapping that carries a set of graph elements, usually the vertices and edges into a set of numbers, usually integers. Many kinds of labeling have been studied and an excellent survey of graph labeling can be found in [3].
Sedlacek [11] introduced the concept of magic labeling. Suppose that $G$ is a graph with $q$ edges. We shall say that $G$ is magic if the edges of $G$ can be labeled by the numbers $1,2,3, \ldots, q$ so that the sum of labels of all the edges incident with any vertex is the same. For concepts of magic type labeling, refer to $[2,12]$.

For a graph $G$ with vertex set $V(G)$ and edge set $E(G)$, an edge-magic total labeling is a bijection $\lambda: V(G) \cup E(G)$ to the set of integers $1,2, \ldots,|V(G) \cup E(G)|$ with the property that, for each edge $x y, \lambda(x)+\lambda(x y)+\lambda(y)=k$, for a fixed integer $k$. Such a labeling is called super if the smallest possible integers are assigned on the vertices. A graph is called edgemagic total if it admits an edge-magic total labeling [2] and super edge-magic total if it admits a super edge-magic total labeling.

The notion of edge-magic total graphs was introduced and studied by Kotzig and Rosa [5] under the name of graphs 
with magic valuation.

Heider's (1946) work on Psychology led Harary (1953) to the notion of signed graphs (or sigraphs in short) and later on Cartwright and Harary (1956) developed the notion of signed digraphs. B. D. Acharya and J. R. Wrens applied the same sigraph theory to balanced Psychology [1] and [13]. Through 4-sigraphs [9] E. Sampathkumar generalized the sigraph and obtained the generalized graph structure [10].

In a sigraph $S=(V, E, \sigma)$, an edge with a positive (negative) sign is called positive edge (negative edge) and $E^{+}(S)\left(E^{-}(S)\right)$ is the set of all positive (negative) edges in the sigraph $S$.

\section{Edge bi-magic total labeling on signed graphs}

Let $S(V, E, \sigma)$ be a signed graph where $V$ is the set of vertices. $E=E^{+} \cup E^{-}$is the disjoint union of the set of positive edges $E^{+}$and the set of negative edges $E^{-}$and $\sigma: E \rightarrow\{+,-\}$ is a map. Let $S G^{(l, m)}$ denote the signed graph with consecutive $l$-positive edges and consecutive $m$-negative edges, where $l+m=q$, the size of the graph. Some concepts of signed graphs can be found in $[4,8,13]$.

\section{Edge bi-magic total labeling on signed necklace}

Definition 3.1. An n-point contact of $K_{2}$ is $N K_{n}$ (i. e) $N K_{n}=$ $K_{2}+n K_{1}$ along with an edge $K_{2}=u v$ is called $a$ necklace with rope $u v$ and is denoted by $N K_{n}$ with rope $u v$.

Definition 3.2. If the edges of a necklace are signed with either + or - then it is called a signed necklace.

Note 3.3. In a signed necklace if $\sigma(u v)=-1$ then it is called a necklace with negative rope and is denoted by $N K_{n}^{-}$.

Note 3.4. In a signed necklace if $\sigma(u v)=1$ then it is called a necklace with positive rope and is denoted by $\mathrm{NK}_{n}^{+}$.

Definition 3.5. $S N K_{(+n-)}^{-}$is signed necklace with a negative rope $u v$ along with $E^{+}=\left\{u w_{1}, u w_{2}, u w_{3}, \ldots, u w_{n}\right\} E^{-}=$ $\left\{w_{1} v, w_{2} v, w_{3} v, \ldots, w_{n} v\right\} \cup\{u v\}$

Definition 3.6. $S N K_{(-n+)}^{+}$is signed necklace with a positive rope $u v$ along with $E^{+}=\left\{w_{1} v, w_{2} v, w_{3} v, \ldots, w_{n} v\right\} \cup\{u v\}$ $E^{-}=\left\{u w_{1}, u w_{2}, u w_{3}, \ldots, u w_{n}\right\}$

Definition 3.7. $S N K_{(-n+)}^{-}$signed necklace with a negative rope uv along with $E^{+}=\left\{w_{1} v, w_{2} v, w_{3} v, \ldots, w_{n} v\right\}$

$E^{-}=\left\{u w_{1}, u w_{2}, u w_{3}, \ldots, u w_{n}\right\} \cup\{u v\}$

Note 3.8. The labeling order in $\mathrm{SNK}_{(+n-)}^{-}$can be adopted for $\mathrm{SNK}_{(-n+)}^{-}$and therefore $\mathrm{SNK}_{(-n+)}^{-}$is called the dual of $\mathrm{SNK}_{(+n-)}^{-}$and vice-versa.

Example:

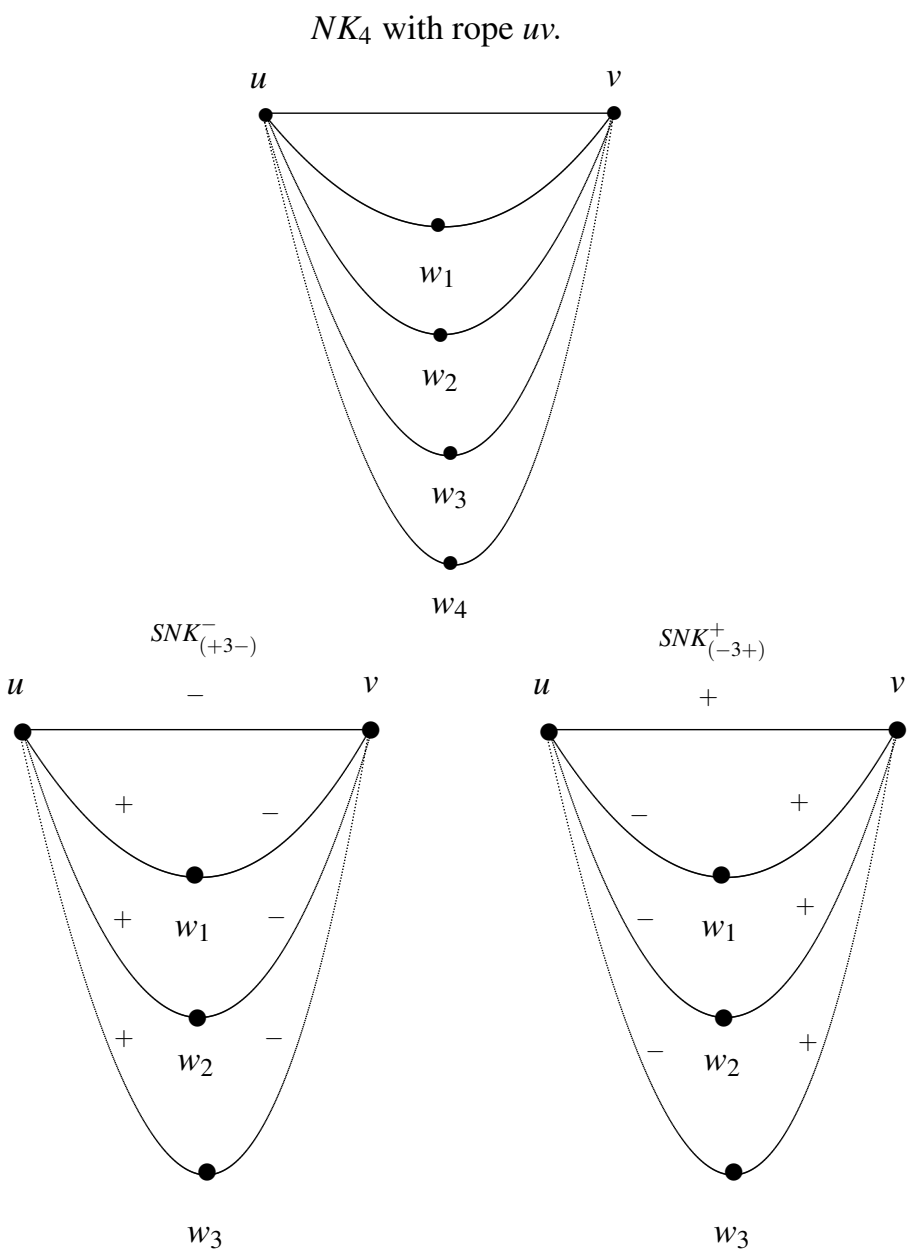

$\mathrm{NK}_{3}$ with negative rope

Definition 3.9. $\mathrm{SNK}_{n}^{+}$is symmetric if $\mathrm{SNK}_{(+n+)}^{+}, \mathrm{SNK}_{(-n-)}^{+}$, $S N K_{(+n-)}^{+}, S N K_{(+n-)}^{-}, S N K_{(-n+)}^{+}$, and $S N K_{(-n+)}^{-}$are the only signed necklaces which are symmetric.

The converse of a sigraph $S=(V, E, \sigma)$ is the sigraph $S^{*}=\left(V, E, \sigma^{*}\right)$, having the same vertex set $V$ and edge set $E$ and $\sigma: E \longrightarrow\{+,-\}$ is defined by $\sigma^{*}(e)=-\sigma(e)$, for all $e \in E=E^{+} \cup E^{-}$.

Note 3.10. $\left(S N K_{(-n+)}^{+}\right)^{*}=\left(S N K_{(+n-)}^{-}\right)$ $\left(\operatorname{SNK}_{(+n-)}^{+}\right)^{*}=\left(\mathrm{SNK}_{(-n+)}^{-}\right)$

Therefore $\left(\left(\operatorname{SNK}_{(-n+)}^{+}\right)^{*}\right)^{*}=\operatorname{SNK}_{(-n+)}^{+}$.

Note 3.11. $\left(S N K_{(+n+)}^{+}\right)^{*}=\left(S N K_{(-n-)}^{-}\right)$ $\left(S N K_{(-n-)}^{-}\right)^{*}=\left(S N K_{(+n+)}^{+}\right)$.

Note 3.12. Dual of $\mathrm{SNK}_{n}$ with positive or negative rope is not its converse.

Note 3.13. Upon the weights, $S\left(S N K_{+n-}^{+}\right) \neq S\left(D S N K_{+n-}^{+}\right)$ $s\left(\operatorname{SNK}_{(+n-)}^{+}\right) \neq s\left(\operatorname{DSNK}_{(+n-)}^{+}\right)$.

In general the positive (negative) upper (lower) strength of a 
signed graph need not be equal to the corresponding positive (negative) upper (lower) strength of its dual.

Definition 3.14. A signed graph which admits an edge bimagic total labeling is called edge bi-magic.

Theorem 3.15. Let $G=S N K_{n}$ be symmetric. Then $G$ is edge bi-magic.

Proof. Consider $S N K_{n}$ with rope $u v . V(G)=\left\{u, v, w_{1}, w_{2}, \ldots, w_{n}\right\} S^{+}=s^{+}=6 n+5$ and $S^{-}=s^{-}=3 n+4$.

$E(G)=\left\{u v,\left\{u w_{1}, u w_{2}, \ldots, u w_{n}, w_{1} v, w_{2} v, \ldots, w_{n} v\right\}\right\}$. Given that $S N K_{n}$ is symmetric.

\section{Case(i):}

Let $S N K_{(+n+)}^{+}$be a signed graph with positive rope $u v$.

Here $V\left(S N K_{(+n+)}^{+}\right)=\left\{u, v, w_{1}, w_{2}, \ldots, w_{n} \cdot\right\}$

$E\left(S N K_{(+n+)}^{+}\right)=\left\{u w_{1}, u w_{2}, \ldots, u w_{n}, u v, w_{1} v, w_{2} v, \ldots, w_{n} v\right\}$

Define $f_{1}: V \cup E^{+} \cup E^{-} \rightarrow\{0,1,2, \ldots, p+q\}$ as follows:

$f_{1}(u)=3 n+3 ; f_{1}(v)=2 n+2$.

$f_{1}\left(w_{i}\right)=3 n+3-i, i=1,2, \ldots, n ; f_{1}(u v)=n$.

$f_{1}\left(u w_{i}\right)=i-1, i=1,2, \ldots, n$.

$f_{1}\left(w_{i} v\right)=n+i, i=1,2, \ldots, n$.

Here $f_{1}$ is a trivial edge bi-magic total labeling where the positive magic weight of $f_{1}$ is $w_{1}^{+}=6 n+5$ and the negative magic weight does not exist.

Hence $S^{+}(G)=6 n+5=s^{+}(G)$.

Note that the label 0 is assigned to an edge and the label $2 n+1$ is omitted and therefore there is no possibility for another nontrivial edge bi-magic total labeling.

\section{Case(ii):}

The bi-magic constants of $S N K_{(+n+)}^{-}$is $S^{+}=s^{+}=6 n+5$ and $S^{-}=s^{-}=4 n+5$.

For:

Assign the same label as in the above case(i) as $f_{2}$. Therefore we get $w_{2}^{+}\left(S N K_{+n+}^{-}\right)=6 n+5, w_{2}^{-}\left(S N K_{+n+}^{-}\right)=4 n+5$. Note that the label 0 assigned to an edge and the label $2 n+1$ is omitted and therefore there is no possibility for another nontrivial edge bi-magic total labeling. Hence $S^{+}=s^{+}=6 n+5$ and $S^{-}=s^{-}=4 n+5$.

\section{Case(iii):}

The bi-magic constants of $S N K_{(-n-)}^{+}$are $S^{+}=s^{+}=6 n+5$ and $S^{-}=s^{-}=4 n+5$. For:

Define $f_{3}: V \cup E^{+} \cup E^{-} \rightarrow\{0,1,2, \ldots, p+q\}$ as follows:

$f_{3}(u)=3 n+3$.

$f_{3}(v)=2 n+2$.

$f_{3}\left(w_{i}\right)=3 n+3-i, i=1,2, \ldots, n$.

$f_{3}(u v)=n$.

$f_{3}\left(u w_{i}\right)=2 n+1-i, i=1,2, \ldots, n$.

$f_{3}\left(w_{i} v\right)=n-i, i=1,2, \ldots, n$.

Here $f_{3}$ is a trivial edge bi-magic total labeling where the positive magic weight of $f_{3}$ is $w_{3}^{+}=6 n+5$ and the negative magic weight of $f_{3}$ is $w_{3}^{-}=4 n+5$.

Case(iv):

The bi-magic constants of $S N K_{(-n-)}^{-}$are $S^{-}=s^{-}=4 n+5$. For:
Assign the same label as in the above case(iii) as $f_{4}$. Therefore we get $w_{4}^{-}\left(S N K_{-n-}^{-}\right)=4 n+5 . w_{4}^{+}$does not exists.

Note that the label 0 assigned to an edge and the label $2 n+1$ is omitted and therefore there is no possibility for another non-trivial edge bi-magic total labeling. Hence $S^{-}=$ $s^{-}=4 n+5$.

\section{Case(v):}

The bi-magic constants of $S N K_{(+n-)}^{+}$are
$S^{+}=s^{+}=6 n+5$ and $S^{-}=s^{-}=3 n+4$.

For:

Define $f_{5}: V \cup E^{+} \cup E^{-} \rightarrow\{0,1,2, \ldots, p+q\}$ as follows:

$f_{5}(u)=3 n+3$.

$f_{5}(v)=2 n+2$.

$f_{5}\left(w_{i}\right)=3 n+3-i, i=1,2, \ldots, n$.

$f_{5}(u v)=n$.

$f_{5}\left(u w_{i}\right)=i-1, i=1,2, \ldots, n$.

$f_{5}\left(w_{i} v\right)=2 n+1-i, i=1,2, \ldots, n$.

Here $f_{5}$ is a trivial edge bi-magic total labeling where the positive magic weight of $f_{5}$ is $w_{5}^{+}=6 n+5$ and the negative magic weight of $f_{5}$ is $w_{5}^{-}=3 n+4$.

\section{Case(vi):}

The bi-magic constants of $S N K_{(+n-)}^{-}$are $S^{+}=s^{+}=7 n+6$ and $S^{-}=s^{-}=4 n+5$.

For:

Define $f_{6}: V \cup E^{+} \cup E^{-} \rightarrow\{0,1,2, \ldots, p+q\}$ as follows:

$f_{6}(u)=3 n+3$.

$f_{6}(v)=2 n+2$.

$f_{6}\left(w_{i}\right)=3 n+3-i, i=1,2, \ldots, n$.

$f_{6}\left(u w_{i}\right)=n+i, i=1,2, \ldots, n$.

$f_{6}\left(w_{i} v\right)=n-i, i=1,2, \ldots, n$.

$f_{6}(u v)=n$.

Here $f_{6}$ is a trivial edge bi-magic total labeling where the positive magic weight of $f_{6}$ is $w_{6}^{+}=7 n+6$ and the negative magic weight of $f_{6}$ is $w_{6}^{-}=4 n+5$.

\section{Case(vii):}

The bi-magic constants of $S N K_{(-n+)}^{+}$are $S^{+}=s^{+}=6 n+5$ and $S^{-}=s^{-}=5 n+6$.

For:

Define $f_{7}: V \cup E^{+} \cup E^{-} \rightarrow\{0,1,2, \ldots, p+q\}$ as follows:

$f_{7}(u)=3 n+3$.

$f_{7}(v)=2 n+2$.

$f_{7}\left(w_{i}\right)=3 n+3-i, i=1,2, \ldots, n$.

$f_{7}(u v)=n$.

$f_{7}\left(u w_{i}\right)=n-i, i=1,2, \ldots, n$.

$f_{7}\left(w_{i} v\right)=n+i, i=1,2, \ldots, n$.

Here $f_{7}$ is a trivial edge bi-magic total labeling where the positive magic weight of $f_{7}$ is $w_{7}^{+}=6 n+5$ and the negative magic weight of $f_{7}$ is $w_{7}^{-}=5 n+6$.

Case(viii):

The bi-magic constants of $S N K_{(-n+)}^{-}$are $S^{+}=s^{+}=5 n+4$ and $S^{-}=s^{-}=4 n+5$.

For:

Define $f_{8}: V \cup E^{+} \cup E^{-} \rightarrow\{0,1,2, \ldots, p+q\}$ as follows: $f_{8}(u)=3 n+3$. 
$f_{8}(v)=2 n+2$.

$f_{8}\left(w_{i}\right)=3 n+3-i, i=1,2, \ldots, n$.

$f_{8}(u v)=n$.

$f_{8}\left(u w_{i}\right)=2 n+1-i, i=1,2, \ldots, n$.

$f_{8}\left(w_{i} v\right)=i-1, i=1,2, \ldots, n$.

Here $f_{8}$ is a trivial edge bi-magic total labeling where the positive magic weight of $f_{8}$ is $w_{8}^{+}=5 n+4$ and the negative magic weight of $f_{8}$ is $w_{8}^{-}=4 n+5$. Hence $S N K_{n}$ is edge bi-magic.

\section{Examples:}
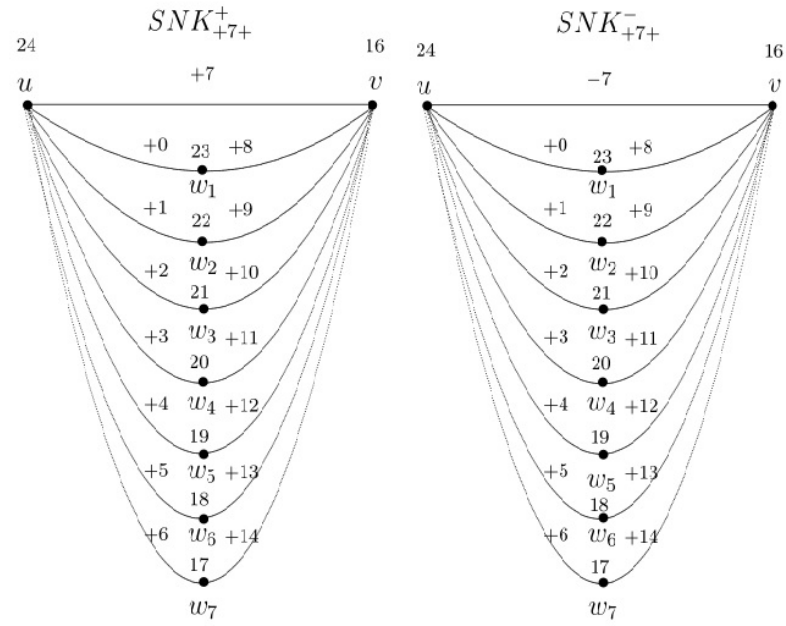

Here $n=7$

Here $n=7$

$S^{+}=s^{+}=47$

$S^{+}=s^{+}=47 ; S^{-}=s^{-}=33$

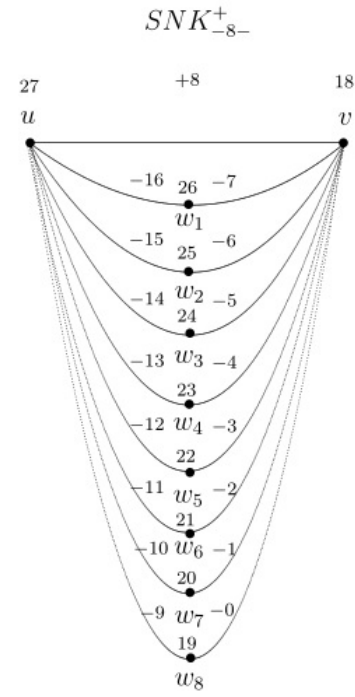

Here $n=8$

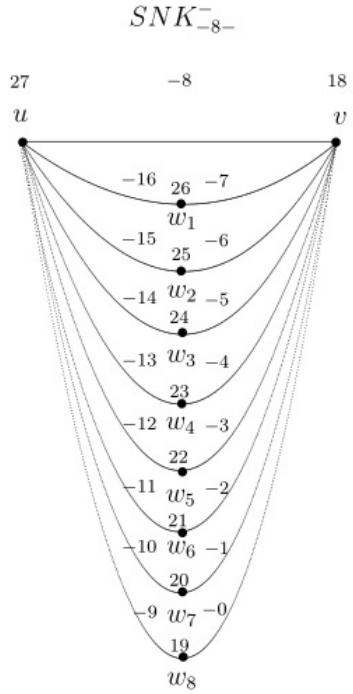

Here $n=8$

$S^{-}=s^{-}=37$

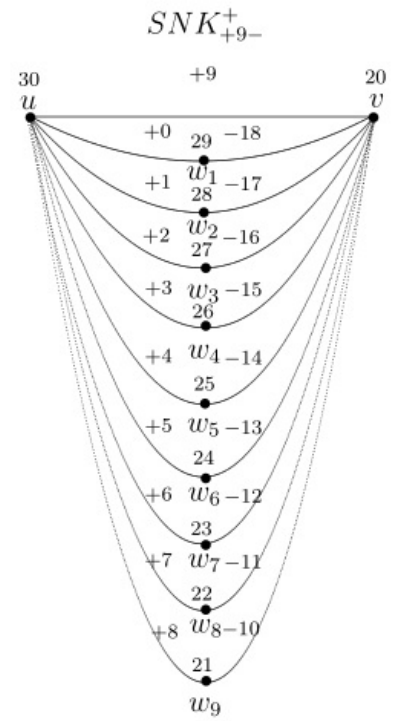

Here $n=9$

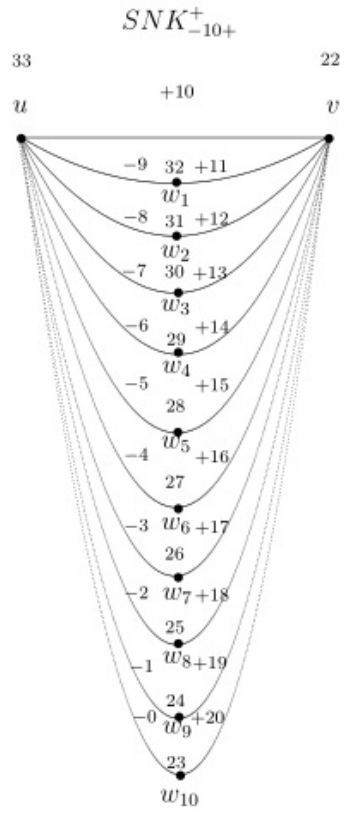

Here $n=10$

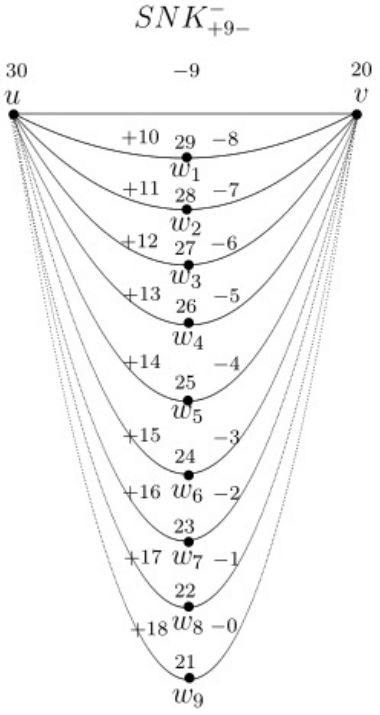

Here $n=9$

$$
S^{+}=s^{+}=59 ; S^{-}=s^{-}=31 \quad S^{+}=s^{+}=69 ; S^{-}=s^{-}=41
$$

$$
S^{+}=s^{+}=65 ; S^{-}=s^{-}=56
$$
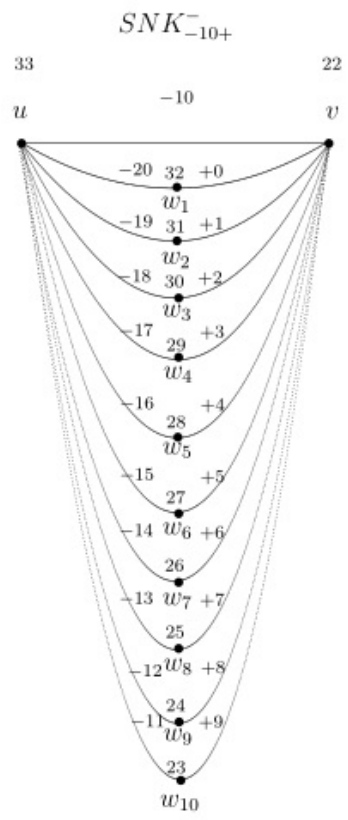

Here $n=10$

$S^{+}=s^{+}=54 ; S^{-}=s^{-}=45$

\section{References}

[1] B. D. Acharya, A chracterization of consistent marked graphs, Nat. Acad. Sci. Letters, 16(12)(1983), 431-440.

[2] Alison M. Marr and W. D. Wallis, Magic Graphs, Birkhauser, Second Edition, 2013.

[3] J. A. Gallian, A dynamic survey of graph labeling, Electron. J. Combin., 19(2013), \# DS6.

[4] M. K. Gill and G. A. Patwarthan, A Characterization of 
Sigraphs which are switching equivalent to their iterated Line graphs, J. Comb. Inf. Syst. Sci.,7-4(1983), 287-296.

[5] A. Kotzig and A. Rosa, Magic valuations of finite graphs, Canad. Math. Bull., 13(1970), 451-461.

[6] MuktiAcharya and Tarkeshwar Singh, Graceful signed graphs, Czechoslovak Math. J., 54(129)(2004), 291-302.

[7] MuktiAcharya,Tarkeshwar Singh, Skolem graceful signed graphs, Util. Math., 82(2010), 97-109.

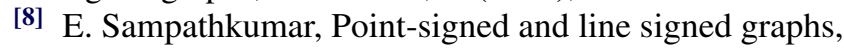
Graph Theory News Letter, 2(2)(1972), 12-17.

[9] E. Sampathkumar, 4-sigraphs, Proceedings of the International Conference on Discrete Mathematics, (ICDM 2006), IISc, Bangalore, 288, 2006.

[10] E. Sampathkumar, Generalized graph structures, Bulletin of Kerala Mathematical Association, 3(2)(2006), 67-123.

[11] J. Sedlacek, Theory of graphs and its applications, Proc. Symposium, 1963, 163-167.

[12] B. M. Stewart, Magic graphs, Canadian J. Math., 18(1966), 1031-1059.

[13] J. R. Weeks, Consensus signed graphs, Siam J. Appl. Math., 36(1)(1979), 1-14.

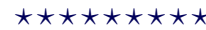 \\ ISSN(P):2319-3786 \\ Malaya Journal of Matematik \\ ISSN(O):2321 - 5666

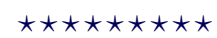

